\title{
Study of $\overline{\mathbf{p}} \rightarrow \bar{\Xi}^{+} \Lambda \mathrm{K}^{-}$with the $\overline{\mathbf{P}} \mathrm{ANDA}$ Detector
}

\author{
Jennifer Pütz ${ }^{1, *}$, Albrecht Gillitzer ${ }^{1}$, James Ritman ${ }^{1}$, and Tobias Stockmanns ${ }^{1}$ on behalf of the PANDA Collaboration \\ ${ }^{1}$ Forschungszentrum Jülich, Wilhelm-Johnen-Straße, 52428 Jülich, Germany
}

\begin{abstract}
For a deeper insight into the mechanisms of non-perturbative QCD it is essential to understand the excitation pattern of baryons. Up to now only the nucleon excitation spectrum has been subject to systematic experimental studies, while very little is known on excited states of double or triple strange baryons.

In studies of antiproton-proton collisions the PANDA experiment is well-suited for a comprehensive baryon spectroscopy program in the multi-strange sector. A large fraction of the inelastic $\bar{p} p$ cross section is associated to final states with a baryon-antibaryon pair together with additional mesons, giving access to excited states both in the baryon and the antibaryon channel.

For final states containing a $\bar{\Xi}^{+} \Xi^{-}$pair, cross sections up to $\mu \mathrm{b}$ are expected, corresponding to production rates of $\sim 10^{6} / \mathrm{d}$ at a luminosity $L=10^{31} \mathrm{~cm}^{-2} \mathrm{~s}^{-1}$. This study focuses on excited $\Xi^{-}$states decaying into $\Lambda K^{-}$. A strategy to reconstruct the reaction $\overline{\mathrm{p}} \mathrm{p} \rightarrow \bar{\Xi}^{+} \Xi^{*}$ and its charge conjugate channel with the $\overline{\mathrm{P} A N D A}$ detector will be presented.
\end{abstract}

\section{Motivation}

In QCD the elementary particles of the strong interaction are quarks and gluons, whereas the minimum quark content of hadrons observable in nature is either three quarks (baryons) or a quark-antiquark pair (mesons). How these composite systems emerge is far less well understood.

To understand the inner structure of baryons and mesons it is necessary to study the excitation pattern of these particles. In baryon spectroscopy one of the main goals is to answer the following questions: Which degrees of freedom are relevant for the excitation mode of baryons? Can they be described by a three-quark or a quark-diquark structure? How important is the dynamics in baryon-meson systems for the generation of resonant baryon states?

Up to now an intense worldwide effort is ongoing to study the nucleon and $\Delta$ excitation spectrum with photo-induced reactions [1-3] while much less information is available on the excitation spectrum of double and triple strange baryons $\Xi$ and $\Omega$. Experimental data on $\Xi$ resonances are coming almost entirely from bubble chamber experiments and not much information was added to PDG [4] since 1988. The study of $\Xi$ baryons will provide independent information on the excitation pattern and allows to verify if the picture deduced from the excited nucleon and $\Delta$ states is correct and has a general validity for baryons in the light quark sector. $\overline{\mathrm{p}}$ collisions provide an ideal entrance channel for baryon spectroscopy in the multi-strange sector since the large cross section allows to collect high statistics data in a reasonable time frame. With the $\bar{P}$ ANDA detector it will be possible to access the excited states of $\bar{\Xi}^{+}$and $\Xi^{-}$directly.

*e-mail: j.puetz@fz-juelich.de

\section{The $\bar{P}$ ANDA Experiment}

The $\overline{\mathrm{P}}$ ANDA experiment is one of the four major projects of FAIR. It has a wide physics program which includes baryon and meson spectroscopy, studies of QCD dynamics, nucleon structure and electromagnetic processes as well as the study of hadrons in nuclei and hypernuclear physics [5].

The PANDA detector consists of two parts, the Target Spectrometer which has a cylindrical geometry and surrounds the interaction point, and the Forward Spectrometer for the reconstruction of high-energy, forward-boosted particles. The modular and flexible design allows the PANDA detector to provide almost $4 \pi$ acceptance for both charged and neutral particles.

One of the properties of hyperons is their long lifetime leading to their typical long decay length and the characteristic topology of the associated particle tracks in the detector. For the reconstruction of these particles a central tracking system is used which consists of the Micro Vertex Detector (MVD), the Straw Tube Tracker (STT) and the Gas Electron Multiplier (GEM) stations. The MVD is the innermost part of the tracking system enclosing the interaction point and is a silicon based high precision vertex detector. The STT consists of gas filled drift tubes arranged in a cylindrical geometry around the beam axis enclosing the interaction point and the MVD. Along the beam line the STT is followed by the GEM stations which are also gas based detectors with planar geometry. The GEM stations are important to reconstruct the trajectories of particles emitted in the forward direction within the Target Spectrometer. The tracking system used in the "Forward Spectrometer" is called Forward Tracking Stations (FTS) and consists of three pairs of tracking stations positioned 
along the beam line. It is based on self-supporting straw tubes which are also used for the STT. The FTS measures the deflection of the particles within a dipole field. Therefore, it is planned to place the first station in front, the second pair within and the third behind the dipole magnet. The different tracking systems will provide a high tracking resolution. In addition, the information from these detectors is combined with other components of $\overline{\mathrm{P}} \mathrm{ANDA}$ to provide a good particle identification.

\section{Study of $\overline{\mathbf{p}} \mathbf{p} \rightarrow \bar{\Xi}^{+} \Lambda \mathrm{K}^{-}$and $\overline{\mathbf{p}} \mathbf{p} \rightarrow \Xi^{-} \bar{\Lambda} \mathrm{K}^{+}$}

\subsection{Event Generation}

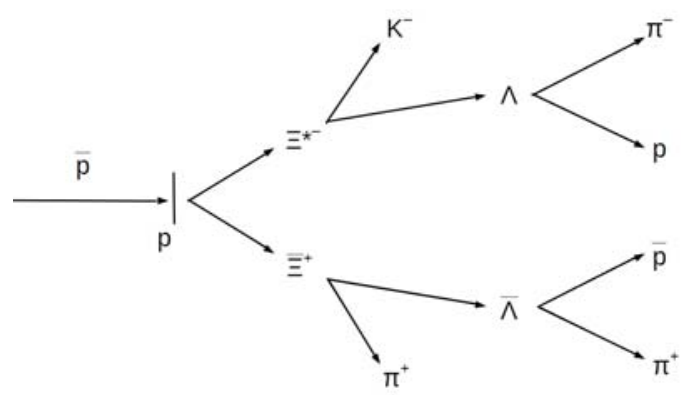

Figure 1. Illustration of the reaction including the decay chain to the detected final state.

For this study 20 million signal events are generated with the event generator EvtGen [6]. The events are generated according to the decay tree shown in Figure 1. Here, the chosen momentum of the antiproton beam is $4.6 \mathrm{GeV} / \mathrm{c}$ which corresponds to a center-of-mass energy of $\sqrt{s}=3.25 \mathrm{GeV}$ and which is about $320 \mathrm{MeV}$ above the production threshold of $\bar{\Xi}^{+} \Lambda \mathrm{K}^{-}$or $\Xi^{-} \bar{\Lambda} \mathrm{K}^{+}$. The given phase space allows the population of the resonant states $\Xi(1690)^{-}$and $\Xi(1820)^{-}$as well as $\bar{\Xi}(1690)^{+}$and $\bar{\Xi}(1820)^{+}$. Table 1 lists the properties of the chosen res-

Table 1. Implemented mass and width of the resonances.

\begin{tabular}{lcc}
\hline State & Mass $\left[\mathrm{MeV} / \mathrm{c}^{2}\right]$ & $\Gamma\left[\mathrm{MeV} / \mathrm{c}^{2}\right]$ \\
\hline$\Xi(1690)^{-}$ & 1690 & 30 \\
$\Xi(1820)^{-}$ & 1823 & 24 \\
\hline
\end{tabular}

onances according to [4]. The fraction between resonant and non-resonant signal which is used as input in EvtGen is shown in Table 2. This assumed ratio is based on the measured total production cross section of both the excited states and ground states of single strange hyperons, see [7].

Since there are neither experimental data nor theoretical predictions for the reaction $\bar{p} p \rightarrow \bar{\Xi}^{+} \Xi^{*-}$, the phase space model (PHSP), generating an isotropic angular distribution, is chosen for each decay mode.

EvtGen does not take into account the curved trajectories of $\bar{\Xi}^{+}$and $\Xi^{-}$in the magnetic field of the solenoid as well as the interaction of both particles with the detector volume. For this reason the propagation of $\bar{\Xi}^{+}$and $\Xi^{-}$is managed by the transport code GEANT4 [8]. As opposed to
Table 2. Decay modes and ratios used as input for the event generation.

\begin{tabular}{lll}
\hline Fraction & Channel & Channel (c.c.) \\
\hline 0.4 & $\bar{\Xi}^{+} \Xi(1690)^{-}$ & $\Xi^{-} \bar{\Xi}(1690)^{+}$ \\
0.4 & $\bar{\Xi}^{+} \Xi(1820)^{-}$ & $\Xi^{-} \bar{\Xi}(1820)^{+}$ \\
0.2 & $\bar{\Xi}^{+} \Lambda \mathrm{K}^{-}$ & $\Xi^{-} \bar{\Lambda} \mathrm{K}^{+}$ \\
\hline
\end{tabular}

$\bar{\Xi}^{+}$and $\Xi^{-}, \bar{\Lambda}$ and $\Lambda$ have various decay modes. Here, the focus is on $\Lambda \rightarrow \mathrm{p}+\pi^{-}$and $\bar{\Lambda} \rightarrow \overline{\mathrm{p}}+\pi^{+}$which have a branching ratio of $\mathrm{BR}\left(\Lambda \rightarrow \mathrm{p}+\pi^{-}\right)=0.634$ [4] each. To avoid the generation of unwanted final states, the branching ratio of $\bar{\Lambda}$ and $\Lambda$ for this decay mode is set to $100 \%$ in GEANT4, respectively. This implies that the final result has to be scaled by the corrected branching ratios.

\subsection{Reconstruction}

The detector parts described in Section 2 measure the momentum of the final state particles, i.e. $\mathrm{p}, \overline{\mathrm{p}}, \mathrm{K}^{-}, \mathrm{K}^{+}$, $\pi^{-}$, and $\pi^{+}$. Therefore, the reconstruction procedure starts with those final state particles and proceeds backwards in the decay chain shown in Figure 1.

The standard tracking algorithms used in PandaRoot [9] - the software framework of PANDA- assume a particle's origin at the interaction point. As already mentioned in Section 2, hyperons have a long decay length and thus decay several centimeters away from the interaction point. This implies, that a reconstruction of the final state particles is not possible with the standard tracking algorithms. Since no realistic algorithm for secondary particles is implemented in PandaRoot, yet, an ideal pattern recognition algorithm is used for the reconstruction of the final state particles. This means, that the hits caused by the charged particles are grouped based on the information of the generated particles. As a consequence, particles with only one hit in any of the tracking detectors will be reconstructed. In order to obtain realistic reconstruction efficiencies, a track filter is used selecting only those candidates which cause at least four hits in either the MVD, STT or GEM detector. This selection criterion is motivated by the trajectory of a charged particle in a homogenous magnetic field. The projection of the trajectory onto, e.g. the x-y-plane (assuming that the particle moves in z-direction), is a circle which is defined by three points in the plane. A fourth hit will confirm the track hypothesis.

Two different selection strategies for composite state particles and the full tree reconstruction are chosen to analyze 10 million events each. The first strategy is a sequential fit procedure in which the candidate selection is based on several cuts on fitted values. In the second case, as the name full decay tree fit implies, the candidate selection is done on the fully reconstructed event. Here, the results of the selection based on the full decay tree fit are presented.

In this analysis no PID information contained in the generated MC event sample is used for reconstructing 
the final state particles. This implies that for a given charge sign each of the corresponding candidates lists, for example $\pi^{+}, \mathrm{K}^{+}$and $\mathrm{p}$, is filled with the same candidates based on all tracks for this charge sign found within the event. The candidate lists then only differ in the mass value assigned to their members based on the respective hypothesis. Since the event selection is completely done on the last stage of the reconstruction, a pre-selection of the events, which requires that the event is reconstructable, in general, is done. Reconstructable means that per event at least three candidates for each charge sign have to be available, otherwise the event is rejected. Table 3 shows the reconstruction efficiency for

Table 3. Reconstruction efficiencies for the final state particles from $\overline{\mathrm{p}} \mathrm{p} \rightarrow \bar{\Xi}^{+} \Lambda \mathrm{K}^{-}$(left) and $\overline{\mathrm{p}} \mathrm{p} \rightarrow \Xi^{-} \bar{\Lambda} \mathrm{K}^{+}$(right). The statistical error is $\sigma \approx 0.1 \%$.

\begin{tabular}{|c|c|c|c|}
\hline type & rec. eff.[\%] & type & rec. eff.[\%] \\
\hline$\pi^{-}$ & 71.2 & $\pi^{+}$ & 70.6 \\
\hline$\pi^{+}(\bar{\Lambda})$ & 68.6 & $\pi^{-}(\Lambda)$ & 68.3 \\
\hline$\pi^{+}\left(\bar{\Xi}^{+}\right)$ & 73.7 & $\pi^{-}\left(\Xi^{-}\right)$ & 73.1 \\
\hline $\mathrm{K}^{-}$(res.) & 84.9 & $\mathrm{~K}^{+}$(res.) & 86.7 \\
\hline $\mathrm{K}^{-}$(cont.) & 85.1 & $\mathrm{~K}^{+}$(cont.) & 86.9 \\
\hline $\mathrm{p}$ & 88.7 & $\mathrm{p}$ & 86.2 \\
\hline$\overline{\mathrm{p}}$ & 82.3 & $\overline{\mathrm{p}}$ & 83.4 \\
\hline
\end{tabular}

the reconstructed final state particles for $\overline{\mathrm{p}} \mathrm{p} \rightarrow \bar{\Xi}^{+} \Lambda \mathrm{K}^{-}$ and $\overline{\mathrm{p}} \mathrm{p} \rightarrow \Xi^{-} \bar{\Lambda} \mathrm{K}^{+}$. Here, the reconstruction efficiency is defined as the number of reconstructed true candidates divided by the number of generated candidates. The results show a lower reconstruction efficiency for $\pi^{-}$ and $\pi^{+}$with respect to $\mathrm{K}^{-}, \mathrm{K}^{+}, \mathrm{p}$ and $\overline{\mathrm{p}}$. There are two reasons for the reduced reconstruction efficiency of the pions. Due to the lifetime of the hyperons, $\pi^{-}$and $\pi^{+}$are produced several centimeters away from the interaction point. Therefore, there are either only a few hits left in the MVD, or none at all. In addition, the track fitting algorithm which uses a Kalman Filter [10] has difficulties to handle low-momentum particles which are produced several centimeters away from the interaction point. In the studied channel, $\pi^{-}$and $\pi^{+}$have a total momentum up to $800 \mathrm{MeV} / \mathrm{c}$ resulting in a loss of reconstructed candidates. After the candidate lists for the final state candidates have been filled, the composite state candidates are combined. To reduce the number of wrongly combined candidates having a mass much higher than the nominal mass of the corresponding hyperon, a mass window cut of $\pm 15 \mathrm{MeV}$ around the nominal hyperon mass is applied. Subsequently, the candidate is passed to the next stage of the reconstruction.

If all particles are reconstructed and combined to a candidate for the full tree, a fit on the full decay tree is applied. For the fit a recursive fitting routine called DecayTreeFitter [11] is used which applies vertex fits as well as kinematic fits with mass constrains for the hyperon masses and a momentum constraint to the initial four-momentum vector of the $\bar{p} p$ system to each candidate. The final selection of the candidate is then done by a cut on the fit probability shown in Figure 2. Candidates

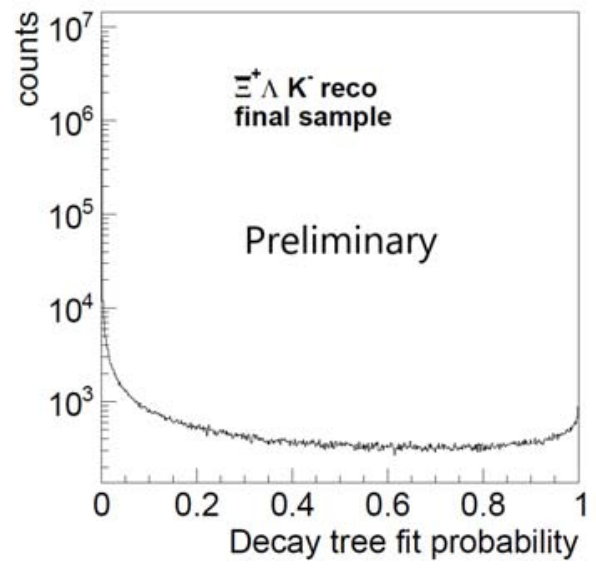

Figure 2. Decay tree fit probability.

with a fit probability less than $0.01 \%$ are rejected. In addition, only candidates for which the fit converged are chosen as final selected candidates. The reconstruction efficiencies achieved for the full reconstruction of the decay tree are $5.4 \%$ for $\bar{\Xi}^{+} \Lambda \mathrm{K}^{-}$and $5.5 \%$ for $\Xi^{-}$ $\bar{\Lambda} \mathrm{K}^{+}$. Both samples have a purity of about $98 \%$. In addition, the fraction of the decay modes is determined to $\sim 38 \%$ for $\bar{\Xi}^{+} \Xi(1690)^{-}, \sim 42 \%$ for $\bar{\Xi}^{+} \Xi(1820)^{-}$, and $\sim 20 \%$ for $\overline{\mathrm{p}} \mathrm{p} \rightarrow \bar{\Xi}^{+} \Lambda \mathrm{K}^{-}$, which is consistent with the input shown in Table 2. Figure 3 shows the Dalitz

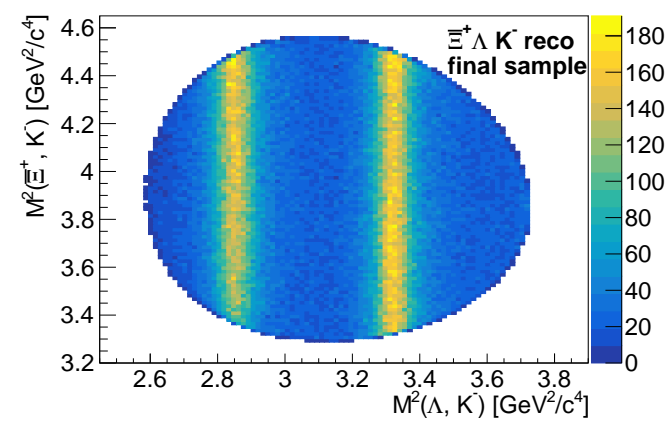

Figure 3. Dalitz plot for the final selected $\bar{\Xi}^{+} \Lambda \mathrm{K}^{-}$candidates. Adapted from [12].

plot for the final reconstructed candidates. Here, the squared mass of the $\Lambda \mathrm{K}^{-}$system is plotted against the squared mass of the $\bar{\Xi}^{+} \mathrm{K}^{-}$system. Both resonances are clearly observable as vertical bands in the Dalitz plot, the $\Xi(1690)^{-}$in the squared mass range between $2.8 \mathrm{GeV}^{2} / \mathrm{c}^{4}$ and $2.95 \mathrm{GeV}^{2} / \mathrm{c}^{4}$ and the $\Xi(1820)^{-}$in the region between $3.25 \mathrm{GeV}^{2} / \mathrm{c}^{4}$ and $3.4 \mathrm{GeV}^{2} / \mathrm{c}^{4}$. The Dalitz plot ratio (Fig. 4) between the MC truth partners of the final reconstructed candidates and the generated candidates appears to be almost flat within the statistical errors which implies that the detector acceptance is structureless for this channel.

Small deviations are visible in the corresponding ratios of the mass distributions for the $\Lambda \mathrm{K}^{-}$and $\bar{\Xi}^{+} \mathrm{K}^{-}$systems. The ratio for the $\Lambda \mathrm{K}^{-}$system (Figure 5) shows a decrease of about $20 \%$ towards lower masses. The reason for this 


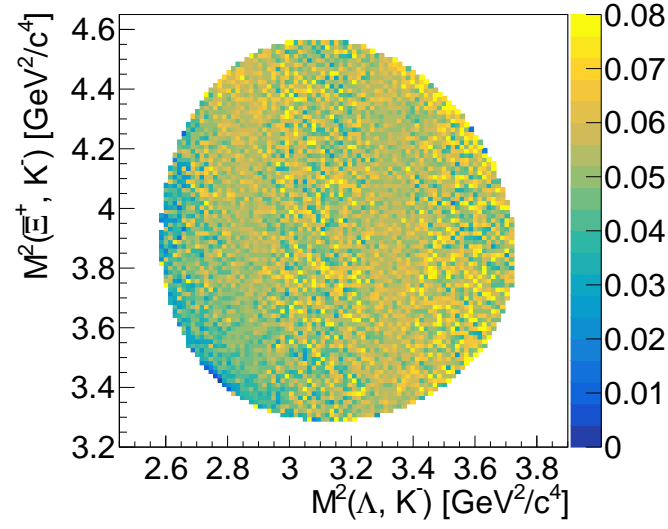

Figure 4. Ratio of the MC truth partner of the final selected and the generated sample. Adapted from [12]

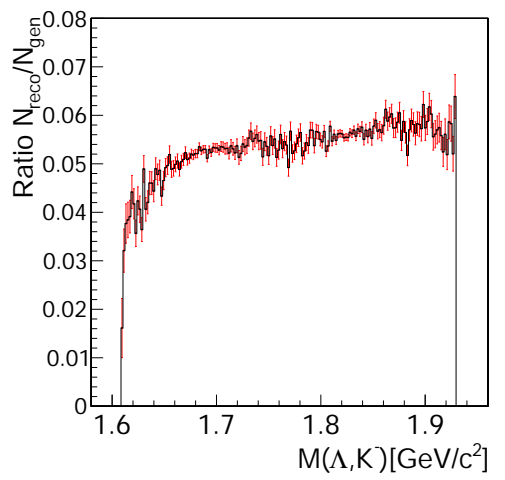

Figure 5. Ratio of the generated and the MC truth partner of the reconstructed $\Lambda \mathrm{K}^{-}$mass distribution. Adapted from [12]

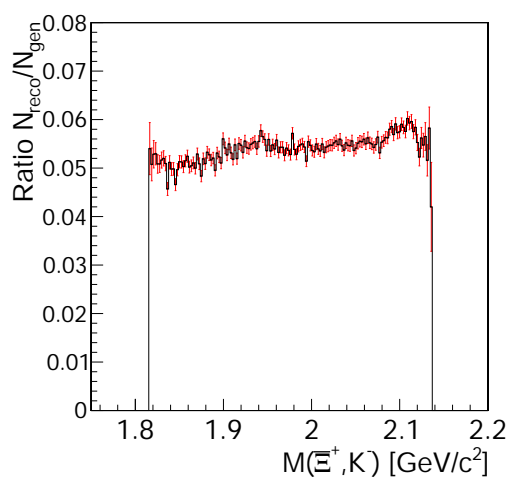

Figure 6. Ratio of the generated and the MC truth partner of the reconstructed $\bar{\Xi}^{+} \mathrm{K}^{-}$mass distribution. Adapted from [12].

behavior has to be explored in more detail based on the angular and momentum distributions of all final state particles. Figure 6 shows the corresponding ratio for the mass distribution for $\bar{\Xi}^{+} \mathrm{K}^{-}$which is within the statistical fluctuation almost flat and varies within $\pm 9 \%$.

To determine the mass and width of the $\Xi$ resonances, two Voigt functions and an additional polynomial function

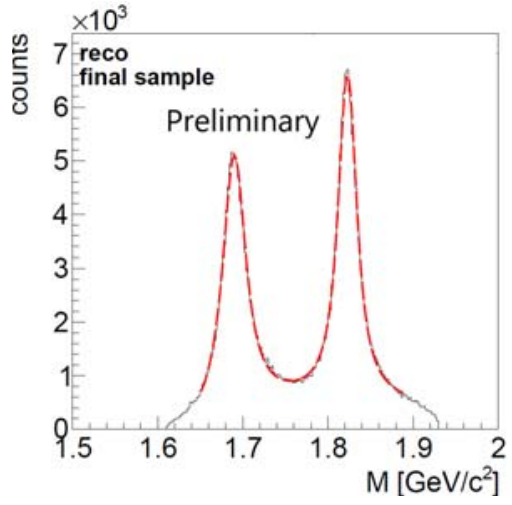

Figure 7. Mass distribution of the final selected $\Lambda \mathrm{K}^{-}$candidates.

are fitted to the mass distribution of the $\Lambda \mathrm{K}^{-}$system, shown in Figure 7. Table 4 summarizes the fit results for the $\Xi^{*}$ and $\bar{\Xi}^{*}$, respectively. The error on the fit values is dominated by systematic uncertainties and is estimated by varying the fit region, systematically. The instrumental width was determined separately by fitting a double Gaussian function to the deviation of the reconstructed and the generated mass distribution. The achieved mass and width for $\Xi(1690)^{-}$and $\bar{\Xi}(1690)^{+}$are in good agreement with the values listed in Table 1. In case of $\Xi(1820)^{-}$and $\bar{\Xi}(1820)^{+}$, the fitted mass values are in good agreement with the input values, while the achieved width differs from the input values by $1.8 \sigma$ for $\Xi(1820)^{-}$and by $2.3 \sigma$ for $\bar{\Xi}(1820)^{+}$, respectively.

Table 4. Fit results for $\Xi^{*}$ and $\bar{\Xi}^{*}$

\begin{tabular}{lcc}
\hline State & Mean $\left[\mathrm{MeV} / \mathrm{c}^{2}\right]$ & $\Gamma\left[\mathrm{MeV} / \mathrm{c}^{2}\right]$ \\
\hline$\Xi(1690)^{-}$ & $1690.0 \pm 0.1$ & $30.1 \pm 0.6$ \\
$\Xi(1820)^{-}$ & $1823.0 \pm 0.1$ & $22.9 \pm 0.4$ \\
$\bar{\Xi}(1690)^{+}$ & $1690.2 \pm 0.1$ & $30.2 \pm 0.6$ \\
$\Xi(1820)^{+}$ & $1823.1 \pm 0.1$ & $22.7 \pm 0.4$ \\
\hline
\end{tabular}

\section{Background Studies}

Beside the investigation of the signal, a study of about 100 million background events at the identical $\overline{\mathrm{p}}$ momentum of $4.6 \mathrm{GeV} / \mathrm{c}$ are generated. The event generation was performed with the Dual Parton Model (DPM) [13] event generator, modeling the inelastic hadronic background in $\overline{\mathrm{p} p}$ collisions. In the analysis these background events were subject to the same selection strategies as used for the signal sample. For both strategies no event out of 100 million generated events was accepted. Assuming that this non-observation of background corresponds to 2.3 events at $90 \%$ confidence level, a lower limit for the signal-to-background ratio can be calculated. The signalto-background ratio is calculated by

$$
\frac{S}{B}=\frac{\sigma_{\mathrm{sig}} \cdot \epsilon_{\mathrm{sig}} \cdot b_{\mathrm{sig}}}{\sigma_{\mathrm{bg}} \cdot \epsilon_{\mathrm{bg}}},
$$

where $\sigma_{\text {sig }}$ is the signal and $\sigma_{\text {bg }}$ the background cross section, $b_{\text {sig }}$ is the total branching fraction of the final selected 
signal evens and $\epsilon_{\text {sig }}$ and $\epsilon_{\mathrm{bg}}$ the reconstruction efficiencies for signal and background, respectively. For the so far unmeasured signal section $\sigma_{\text {sig }}=1 \mu \mathrm{b}$ is assumed. This assumption is based on the measured total production cross sections of $\Xi^{-} \bar{\Xi}^{+}$production [7] and on the production of excited states and ground states of single strange hyperons [14] in $\bar{p} p$ collisions. Furthermore, the inelastic cross section for $\bar{p} p$ at a beam momentum of $4.6 \mathrm{GeV} / \mathrm{c}$ is $\sigma_{\text {bg }}=50 \mathrm{mb}$ [4].

As already discussed in Section 3.1, the branching ratio for $\Lambda$ into $\mathrm{p}$ and $\pi^{-}$as well as for $\bar{\Lambda}$ into $\overline{\mathrm{p}}$ and $\pi^{+}$was set to $100 \%$. This is corrected in the following by taking the correct branching ratio $b_{\text {sig }}=b_{\wedge}^{2}=0.483$ for the final selected signal into account. The reconstruction efficiency achieved with the full decay tree fit is $\epsilon_{\text {sig }}=5.4 \%$ for $\bar{\Xi}^{+}$ $\Lambda \mathrm{K}^{-}$and $\epsilon_{\text {sig }}=5.5 \%$ for $\Xi^{-} \bar{\Lambda} \mathrm{K}^{+}$. The reconstruction efficiency for the background events at $90 \%$ confidence level is $\epsilon_{\mathrm{bg}}=2.3 \cdot 10^{-8}$. The resulting lower limits for the signal-to-background ratio are listed in Table 5.

Table 5. Signal-to-Background ratio.

\begin{tabular}{lc}
\hline & $S / B$ \\
\hline $\bar{\Xi}^{+} \Lambda \mathrm{K}^{-}$ & $>19.1$ \\
$\Xi^{-} \bar{\Lambda} \mathrm{K}^{+}$ & $>19.5$ \\
\hline
\end{tabular}

\section{Summary}

The study of in total 20 million signal events for the process $\bar{p} p \rightarrow \bar{\Xi}^{+} \Xi^{*}$ where $\Xi^{*} \rightarrow \Lambda \mathrm{K}^{-}$as well as for the charge conjugate channel is presented. Here, for the signal, two $\Xi$ resonances $\Xi(1690)^{-}$and $\Xi(1820)^{-}$as well as their charge conjugate were chosen. For this analysis no Monte Carlo information is used to assign the particle type to the reconstructed candidates. With the full decay tree fit a reconstruction efficiency of $5.4 \%$ was achieved for $\bar{\Xi}^{+} \Lambda$ $\mathrm{K}^{-}$and $5.5 \%$ for $\Xi^{-} \bar{\Lambda} \mathrm{K}^{+}$, and the purity for both samples is about $98 \%$. The reconstructed fraction of the resonant and non-resonant contribution is consistent with the input. Assuming the luminosity to be $L=10^{31} \mathrm{~cm}^{-2} \mathrm{~s}^{-1}$, the reconstruction efficiency corresponds to at least 18,000 fully reconstructed events per day.

The comparison between the reconstructed values for the masses and widths of the $\Xi$ resonances with the input, shows that the masses for both resonances are in good agreement with the input. In case of the $\Xi(1690)^{-}$, also the width is in good agreement with the input while the widths for the $\Xi(1820)^{-}$and its charge conjugate partner differ by about two standard deviations from the input value.

About 100 million events generated with the DPM generator are studied with the same analysis strategies as applied to the signal events. This study of background events shows that the hadronic background is sufficiently suppressed since no event out of 100 million generated events was accepted. The usage of open PID does not reduce the purity and the signal-to-background ratio significantly.

\section{Outlook}

This first study, although still based on some simplifications, shows already the promising perspective of investigating $\Xi$ resonances with $\bar{P}$ ANDA. One of these simplifications is the usage of an ideal tracking algorithm, as long as a realistic tracking algorithm is not yet available. As soon as a tracking algorithm for secondary particles will be available this study should be redone.

Another point is that in the analysis with the sequential fit procedure ideal particle identification (PID) was used which leads to the fact, that the results cannot be directly compared. Therefore, the analysis should be redone with open PID.

It is already mentioned, that the angular distribution of the reaction $\overline{\mathrm{p} p} \rightarrow \bar{\Xi}^{+} \Xi^{*}$ is unknown. Therefore, an isotropic angular distribution is used for the event generation. This simplification should be replaced by a more realistic approach based on future theoretical studies.

As discussed in Section 1 the information on $\Xi$ resonances is poor. In case of both $\Xi(1690)^{-}$and $\Xi(1820)^{-}$, only tentative assignments of spin and parity are available. In order to explore the feasibility of determining these quantum numbers, a partial wave analysis of the reaction $\bar{p} p \rightarrow$ $\bar{\Xi}^{+} \Lambda \mathrm{K}^{-}$and $\overline{\mathrm{p}} \mathrm{p} \rightarrow \Xi^{-} \bar{\Lambda} \mathrm{K}^{+}$is required. First studies in this respect are already ongoing.

\section{References}

[1] The Electron Stretcher Accelerator (ELSA), https://www-elsa.physik.uni-bonn.de/elsa-facility_en. html

[2] The Mainz Microtron (MAMI), https://www.blogs.uni-mainz.de/fb08-nuclear-physics/ accelerators-mami-mesa/the-mainz-microtron

[3] Thomas Jefferson National Accelerator Facility (JLab), https://www.jlab.org

[4] M. Tanabashi, et al. (Particle Data Group), Phys. Rev. D 98, 030001 (2018)

[5] W. Erni, et al., arXiv preprint, arXiv:0903.3905 (2009)

[6] D. J. Lange, Nucl. Instr. Meth. A 462, 152 (2001)

[7] V. Flaminio, et al., CERN-HERA-84-01 (1984)

[8] S. Agostinelli, et al., Nucl. Instr. and Meth. in Phys. Res. A 506, 250-303 (2003)

[9] S. Spataro, Journal of Physics: Conference Series 331, 032031 (2011)

[10] A. Fontana et al., Journal of Physics: Conference Series 119, 032018 (2008)

[11] W. D. Hulsbergen, Nucl. Instr. and Meth. in Phys. Res. A 552, 566-575 (2005)

[12] J. Pütz, PhD Thesis, Rheinische Friedrich-WilhelmsUniversität Bonn (2020)

[13] A. Capella, NATO ASI Series B: Physics 333, 287310 (1994)

[14] B. Musgrave et al., Il Nuovo Cimento 35, 735 (1965) 